\title{
MIXED STRUCTURES ON A MANIFOLD WITH BOUNDARY
}

\author{
ALBERTO CAVICCHIOLI \\ Dipartimento di Matematica, Università di Modena e Reggio Emilia, Via Campi 213/B, \\ 41100 Modena, Italia \\ e-mail: cavicchioli.alberto@unimo.it \\ YURIJ V. MURANOV \\ Department of Information Science and Management, Institute of Modern Knowledge, \\ ulica Gor'kogo 42, 210004 Vitebsk, Belarus \\ e-mail:ymuranov@imk.edu.by \\ and FULVIA SPAGGIARI \\ Dipartimento di Matematica, Università di Modena e Reggio Emilia, Via Campi 213/B, \\ 41100 Modena, Italia \\ e-mail: spaggiari.fulvia@unimo.it
}

(Received 19 July, 2005; accepted 8 September, 2005)

\begin{abstract}
For a closed topological $n$-manifold $X$, the surgery exact sequence contains the set of manifold structures and the set of tangential structures of $X$. In the case of a compact topological $n$-manifold with boundary $(X, \partial X)$, the classical surgery theory usually considers two different types of structures. The first one concerns structures whose restrictions are fixed on the boundary. The second one uses two similar structures on the manifold pair. In his classical book, Wall mentioned the possibility of introducing a mixed type of structure on a manifold with boundary. Following this suggestion, we introduce mixed structures on a topological manifold with boundary, and describe their properties. Then we obtain connections between these structures and the classical ones, and prove that they fit in some surgery exact sequences. The relationships can be described by using certain braids of exact sequences. Finally, we discuss explicitly several geometric examples.
\end{abstract}

2000 Mathematics Subject Classification. Primary 57R67, 57Q10 Secondary 57R10, 55U35, 18F25.

1. Introduction. Let $X$ be a closed topological manifold of dimension $n$. Let $\mathcal{S}(X)=\mathcal{S}^{s}(X)$ denote the set of equivalence classes of simple homotopy equivalences $f: M \rightarrow X$, where $M$ is a compact topological $n$-dimensional manifold. The equivalence relation is given by the usual $s$-cobordism of such maps (see [14], [15], and [24]). It is well-known that for $n \geq 5$ the set $\mathcal{S}(X)$ fits into the surgery exact

Partially supported by the Russian Foundation for Fundamental Research Grant No. 05-01-00993, by the GNSAGA of the National Research Council of Italy, by the MIUR (Ministero della Istruzione, Università e Ricerca) of Italy within the project Proprietà Geometriche delle Varietà Reali e Complesse, and by a Research grant of the University of Modena and Reggio Emilia. 
sequence (see, for example, [15] and [24, $§ 10]$ )

$$
\cdots \rightarrow L_{n+1}\left(\pi_{1}(X)\right) \rightarrow \mathcal{S}(X) \rightarrow[X, G / T O P] \rightarrow L_{n}\left(\pi_{1}(X)\right) \rightarrow \cdots
$$

where $L_{*}$ denotes the surgery obstruction groups. In fact, the set of homotopy classes $[X, G / T O P]$ coincides with the set of equivalence classes $\mathcal{T}(X)$ of tangential structures on the manifold $X$ (see [15] and [24]).

Given a compact topological $n$-manifold with boundary $(X, \partial X)$, we can consider a set of manifold structures $\mathcal{S}^{\partial}(X, \partial X)$ and a set of tangential structures $\mathcal{T}^{\partial}(X, \partial X)$ on $X$, where the structures are fixed on the boundary $\partial X$. For $n \geq 5$ these sets fit in the surgery exact sequence of a bordered manifold with fixed structures on its boundary

$$
\cdots \rightarrow L_{n+1}\left(\pi_{1}(X)\right) \rightarrow \mathcal{S}^{\partial}(X, \partial X) \rightarrow \mathcal{T}^{\partial}(X, \partial X) \rightarrow L_{n}\left(\pi_{1}(X)\right) \rightarrow \cdots
$$

A third type of structure is given by a set of manifold structures and a set of tangential structures on the pair $(X, \partial X)$. For $n \geq 5$ these structures fit in a surgery exact sequence of relative groups (see [15] and [24])

$$
\rightarrow L_{n+1}\left(\pi_{1}(\partial X) \rightarrow \pi_{1}(X)\right) \rightarrow \mathcal{S}(X, \partial X) \rightarrow \mathcal{T}(X, \partial X) \rightarrow L_{n}\left(\pi_{1}(\partial X) \rightarrow \pi_{1}(X)\right) .
$$

Recent results on various types of relative groups which arise naturally in surgery theory were obtained in [4] and [5]. This permits the investigation of the splitting obstruction groups and the surgery obstruction groups for a manifold pair and for a triple of manifolds, obtaining explicit computations in many cases (see [3], [12], [13], and [20]).

Following the classical book of Wall (see [24, p. 116]), we introduce a mixed type of structure on a pair $(X, \partial X)$, where $X$ is a compact topological $n$-manifold. We deal with a set of tangential structures on $X$ whose restrictions on the boundary give a set of manifold structures on $\partial X$ together with the corresponding equivalence relations. We shall denote such structures by $\mathcal{T} \mathcal{S}(X, \partial X)$.

By using the realizations of exact sequences (1.1)-(1.3) on the spectra level (see [2], [3], [5], [14] and [15]) we obtain a spectrum $\mathbb{T S}(X, \partial X)$ with homotopy groups

$$
\pi_{i}(\mathbb{\mathbb { S } S}(X, \partial X))=\mathcal{T} \mathcal{S}_{i}(X, \partial X)
$$

for which there exists an isomorphism

$$
\mathcal{T} \mathcal{S}_{n+1}(X, \partial X) \cong \mathcal{T} \mathcal{S}(X, \partial X) .
$$

So the set $\mathcal{T} \mathcal{S}(X, \partial X)$ is a group. Then we study the relationships between this group and the algebraic version of exact sequences (1.1)-(1.3). The connections are given by certain braids of exact sequences shown in the statement of Theorem 6 (see Section 3). In Section 2 we recall some necessary definitions and results of Ranicki on algebraic surgery exact sequences and applications of spectra to $L$-theory. In Section 3 we define mixed structures on a manifold with boundary, and describe their properties. This section contains the main theorem of the paper. In Section 4 we discuss some applications of our results, also considering several geometrical examples for which explicit computations are obtained.

2. Preliminaries. First we recall some basic facts about the application of homotopy category of spectra to surgery theory (see [2]-[5], [14], and [23]). A spectrum 
$\mathbb{E}$ consists of a collection of $C W$-complexes $\left\{\left(E_{n}, *\right)\right\}, n \in \mathbb{Z}$, together with a collection of cellular maps $\left\{\epsilon_{n}: S E_{n} \rightarrow E_{n+1}\right\}$, where $S E_{n}$ denotes the suspension of the space $E_{n}$. A spectrum $\mathbb{E}$ is called an $\Omega$-spectrum if the adjoint maps $\epsilon_{n}^{\prime}: E_{n} \rightarrow \Omega E_{n+1}$ are homotopy equivalences, for every $n \in \mathbb{Z}$ (see [23]). Given a spectrum $\mathbb{E}$, the spectrum $\Sigma \mathbb{E}$ with

$$
\{\Sigma \mathbb{E}\}_{n}=\mathbb{E}_{n+1}, \quad\{\Sigma \epsilon\}_{n}=\epsilon_{n+1}
$$

is defined. The functor $\Sigma$ has an inverse functor $\Sigma^{-1}$. Iterated functors $\Sigma^{k}, k \in \mathbb{Z}$, on the category of spectra are also defined. According to [23] there is an isomorphism of homotopy groups $\pi_{n}(\mathbb{E})=\pi_{n+k}\left(\Sigma^{k} \mathbb{E}\right)$, for every spectrum $\mathbb{E}$. A homotopy commutative square of spectra

$$
\begin{array}{ll}
\mathbb{G} & \rightarrow \mathbb{H} \\
\downarrow & \downarrow \\
\mathbb{E} & \rightarrow \mathbb{F}
\end{array}
$$

is a pull-back square if and only if the fibres of the parallel maps are naturally homotopy equivalent. Square (2.1) is a push-out square if and only if the cofibers of the parallel maps are naturally homotopy equivalent (see [23]). In the homotopy category of spectra the concepts of pull-back and push-out squares are equivalent.

Recall that the surgery obstruction groups $L_{n}(\pi)$ are defined for every group $\pi$ with an orientation homomorphism $w: \pi \rightarrow\{ \pm 1\}$. For brevity, we shall not include orientation homomorphisms in our notation, and assume that all homomorphisms of groups agree with the corresponding orientation homomorphisms. For every oriented group $\pi$ there exists a $\Omega$-spectrum $\mathbb{L}(\pi)$ with homotopy groups $\pi_{n}(\mathbb{L}(\pi))=L_{n}(\pi)$. A homomorphism $f: \pi \rightarrow \pi^{\prime}$ of oriented groups induces a cofibration of $\Omega$-spectra

$$
\mathbb{L}(\pi) \longrightarrow \mathbb{L}\left(\pi^{\prime}\right) \longrightarrow \mathbb{L}(f)
$$

with a homotopy long exact sequence

$$
\cdots \rightarrow L_{n}(\pi) \rightarrow L_{n}\left(\pi^{\prime}\right) \rightarrow L_{n}(f) \rightarrow L_{n-1}(\pi) \rightarrow \cdots
$$

Now we recall the definition of a topological normal map into a closed topological manifold $X$ of dimension $n$ (see for example [15]). A topological normal map (or, equivalently, a $t$-triangulation of $X$ )

$$
(f, b): M \rightarrow X
$$

is given by the following conditions:

(i) an $n$-dimensional manifold $M$ with a normal topological block bundle

$$
\begin{array}{r}
v_{M}=v_{M \subset S^{n+k}}: M \rightarrow \operatorname{BTOP}(k), \\
\rho_{M}: S^{n+k} \rightarrow S^{n+k} / \overline{S^{n+k}-E\left(v_{M}\right)}=T\left(v_{M}\right)
\end{array}
$$

(ii) an $n$-dimensional manifold $X$ with a topological block bundle

$$
\begin{gathered}
v_{X}: X \rightarrow B T O P(k), \\
\rho_{X}: S^{n+k} \rightarrow T\left(v_{X}\right) ;
\end{gathered}
$$


(iii) a degree one $\operatorname{map} f: M \rightarrow X$;

(iv) a map of topological block bundles $b: v_{M} \rightarrow v_{X}$, covering $f$, such that

$$
T(b)_{*}\left(\rho_{M}\right)=\rho_{X} \in \pi_{n+k}\left(T\left(v_{X}\right)\right) .
$$

The topological normal structure set $\mathcal{T}(X)$ of the manifold $X$ is the set of concordance classes of normal maps $(f, b): M \rightarrow X$. For $n \geq 5$, it coincides with the set $[X, G / T O P]$ (see [15]). Recall that a concordance of topological normal maps

$$
\left(f_{i}, b_{i}\right): M_{i} \rightarrow X, \quad i=0,1
$$

is given by a topological normal map of triads

$$
\left((g, c) ;\left(f_{0}, b_{0}\right),\left(f_{1}, b_{1}\right)\right):\left(W ; M_{0}, M_{1}\right) \rightarrow(X \times I ; X \times\{0\}, X \times\{1\}),
$$

where $I=[0,1]$ and $W$ is a compact $(n+1)$-dimensional topological manifold with boundary $\partial W=M_{0} \cup M_{1}$ (see $\left.[\mathbf{1 5}, \S 7.1]\right)$.

Let $X$ be a closed topological $n$-dimensional manifold. An $s$-triangulation of $X$ is a simple homotopy equivalence $f: M^{n} \rightarrow X$, where $M^{n}$ is a closed topological $n$-dimensional manifold. The set of concordance classes of $s$-triangulations of $X$ is denoted by $\mathcal{S}(X)$ (see [14], [15], and [24]). Recall that a concordance between two $s$-triangulations

$$
\left(f_{i}, b_{i}\right): M_{i} \rightarrow X, \quad i=0,1
$$

is given by a simple homotopy equivalence of triads

$$
\left(g ; f_{0}, f_{1}\right):\left(W ; M_{0}, M_{1}\right) \rightarrow(X \times I ; X \times\{0\}, X \times\{1\}),
$$

where $W$ is a compact $(n+1)$-dimensional topological manifold with boundary $\partial W=$ $M_{0} \cup M_{1}(\operatorname{see}[15, \S 7.1])$.

Now let $(X, \partial X)$ be a compact $n$-dimensional topological manifold with boundary $\partial X$. Let us consider the case of structures on $X($ rel $\partial)$ for which a manifold structure on the boundary $\partial X$ is fixed. A topological normal map of pairs (see $[\mathbf{1 5}, \S 7.1]$ )

$$
((f, b),(\partial f, \partial b)):\left(M^{n}, \partial M\right) \rightarrow(X, \partial X)
$$

together with a homeomorphism $\partial f: \partial M \rightarrow \partial X$ is called a $t_{\partial}$-triangulation of the compact $n$-dimensional manifold $X$. The set of concordance classes of $t_{\partial}$-triangulations is denoted by $\mathcal{T}^{\partial}(X, \partial X)$ (see $[\mathbf{1 5}, \S 7.1]$ and [24, $\left.\S 10\right]$ ). Recall that a concordance of $t_{\partial}$-triangulations

$$
\left(\left(f_{i}, b_{i}\right),\left(\partial f_{i}, \partial b_{i}\right)\right):\left(M_{i}, \partial M_{i}\right) \rightarrow(X, \partial X), \quad i=0,1
$$

is given by a topological normal map of 4-ads

$\left((h, d) ;(g, c),\left(f_{0}, b_{0}\right),\left(f_{1}, b_{1}\right)\right):\left(W ; V, M_{0}, M_{1}\right) \rightarrow(X \times I ; \partial X \times I, \partial X \times\{0\}, \partial X \times\{1\})$

where

$$
V=\partial M_{0} \times I, \quad \partial V=\partial M_{0} \cup \partial M_{1}
$$


and

$$
(g, c)=\partial f_{0} \times I d: V \rightarrow \partial X \times I .
$$

An $s_{\partial}$-triangulation of a compact $n$-dimensional manifold $X$ with boundary $\partial X$ is a simple homotopy equivalence of pairs (see $[\mathbf{1 5}, \S 7.1])$

$$
(f, \partial f):\left(M^{n}, \partial M\right) \rightarrow(X, \partial X)
$$

together with a homeomorphism $\partial f: \partial M \rightarrow \partial X$. The set of concordance classes of $s_{\partial}$-triangulations is denoted by $\mathcal{S}^{\partial}(X, \partial X)$ (see $[\mathbf{1 5}, \S 7.1]$ and $\left.[\mathbf{2 4}, \S 10]\right)$. Recall that a concordance of two $s_{\partial}$-triangulations

$$
\left(f_{i}, \partial f_{i}\right):\left(M_{i}, \partial M_{i}\right) \rightarrow(X, \partial X), \quad i=0,1
$$

is given by a simple homotopy equivalence of 4 -ads

$$
\left(h ; g, f_{0}, f_{1}\right):\left(W ; V, M_{0}, M_{1}\right) \rightarrow(X \times I ; \partial X \times I, \partial X \times\{0\}, \partial X \times\{1\})
$$

where

$$
V=\partial M_{0} \times I, \quad \partial V=\partial M_{0} \cup \partial M_{1}
$$

and

$$
g=\partial f_{0} \times I d: V \rightarrow \partial X \times I .
$$

Let $X$ be a compact $n$-dimensional topological manifold with boundary $\partial X$. A $t$-triangulation of $(X, \partial X)$ is a topological normal map of pairs (see $[\mathbf{1 5}, \S 7.1])$

$$
((f, b),(\partial f, \partial b)):\left(M^{n}, \partial M\right) \rightarrow(X, \partial X)
$$

where $M$ is a compact $n$-dimensional topological manifold with boundary $\partial M$. The set of concordance classes of $t$-triangulations is denoted by $\mathcal{T}(X, \partial X)$ (see $[15, \S 7.1]$ and $[\mathbf{1 7}, \S 10])$. Recall that a concordance of $t$-triangulations

$$
\left(\left(f_{i}, b_{i}\right),\left(\partial f_{i}, \partial b_{i}\right)\right):\left(M_{i}, \partial M_{i}\right) \rightarrow(X, \partial X), \quad i=0,1
$$

is given by a topological normal map of 4-ads

$\left((h, d) ;(g, c),\left(f_{0}, b_{0}\right),\left(f_{1}, b_{1}\right)\right):\left(W ; V, M_{0}, M_{1}\right) \rightarrow(X \times I ; \partial X \times I, \partial X \times\{0\}, \partial X \times\{1\})$ where

$$
\partial V=\partial M_{0} \cup \partial M_{1}
$$

An $s$-triangulation of $(X, \partial X)$ is a simple homotopy equivalence of pairs

$$
(f, \partial f):(M, \partial M) \rightarrow(X, \partial X)
$$

where $M$ is a compact $n$-dimensional topological manifold with boundary $\partial M$ (see $[15, \S 7.1])$. The set of concordance classes of $s$-triangulations of $(X, \partial X)$ is denoted by $\mathcal{S}(X, \partial X)($ see $[15, \S 7.1]$ and $[\mathbf{1 7}, \S 10])$. 
Recall that a concordance of two s-triangulations

$$
\left(f_{i}, \partial f_{i}\right):\left(M_{i}, \partial M_{i}\right) \rightarrow(X, \partial X), \quad i=0,1
$$

is given by a simple homotopy equivalence of 4 -ads

$$
\left(h ; g, f_{0}, f_{1}\right):\left(W ; V, M_{0}, M_{1}\right) \rightarrow(X \times I ; \partial X \times I, \partial X \times\{0\}, \partial X \times\{1\})
$$

where

$$
\partial V=\partial M_{0} \cup \partial M_{1}
$$

For every topological space $X$ there exists an algebraic surgery exact sequence (see [14] and [15])

$$
\cdots \rightarrow L_{n+1}\left(\pi_{1}(X)\right) \rightarrow \mathcal{S}_{n+1}(X) \rightarrow H_{n}\left(X ; \mathbf{L}_{\bullet}\right) \rightarrow L_{n}\left(\pi_{1}(X)\right) \rightarrow \cdots
$$

where $\mathbf{L}_{\mathbf{0}}$ is the 1 -connected cover of the simply connected surgery $\Omega$-spectrum $\mathbf{L}_{\bullet}(\mathbb{Z})$ with $\mathbf{L}_{\bullet 0} \simeq G / T O P$. This algebraic surgery sequence is a homotopy long exact sequence of the cofibration

$$
X_{+} \wedge \mathbf{L}_{\bullet} \rightarrow \mathbb{L}\left(\pi_{1}(X)\right)
$$

By definition, we have $\mathcal{S}_{i}(X)=\pi_{i}(\mathbb{S}(X))$ for a homotopy cofiber $\mathbb{S}(X)$ of the map in (2.5). If $X$ is a closed $n$-dimensional topological manifold, then we have

$$
\pi_{n+1}(S(X))=\mathcal{S}_{n+1}(X) \cong \mathcal{S}(X)
$$

and sequence (1.1) is isomorphic to the left part (for $m \geq n$ ) of the algebraic surgery exact sequence in (2.4). For the case of a compact topological manifold $(X, \partial X)$ with boundary, we have a spectrum $\mathbb{S}^{\partial}(X, \partial X)$ with homotopy groups

$$
\pi_{i}\left(\mathbb{S}^{\partial}(X, \partial X)\right)=\mathcal{S}_{i}^{\partial}(X, \partial X)
$$

and

$$
\mathcal{S}_{n+1}^{\partial}(X, \partial X) \cong \mathcal{S}(X, \partial X), \quad H_{n}\left(X ; \mathbf{L}_{\bullet}\right) \cong \mathcal{T}^{\partial}(X, \partial X) .
$$

Furthermore, a similar situation holds for exact sequences (1.2) and (1.3) (see [14] and [15]). More precisely, there exist $\Omega$-spectra $\mathbb{S}(X, \partial X)$ and $\mathbb{S}^{\partial}(X, \partial X)$ with homotopy groups

$$
\pi_{i}\left(\mathbb{S}^{\partial}(X, \partial X)\right)=\mathcal{S}_{i}^{\partial}(X, \partial X), \quad \pi_{i}(\mathbb{S}(X, \partial X))=\mathcal{S}_{i}(X, \partial X)
$$

for which there are isomorphisms

$$
\mathcal{S}_{n+1}^{\partial}(X, \partial X) \cong \mathcal{S}^{\partial}(X, \partial X), \quad \mathcal{S}_{n+1}(X, \partial X) \cong \mathcal{S}(X, \partial X) .
$$

For the tangential structures in exact sequences (1.1) and (1.2), we have

$$
\mathcal{T}(X, \partial X) \cong H_{n}\left(X, \partial X ; \mathbf{L}_{\bullet}\right), \quad \mathcal{T}^{\partial}(X, \partial X) \cong H_{n}\left(X ; \mathbf{L}_{\bullet}\right) .
$$


It follows from [14], [15], and [23] that there exists the following commutative diagram of exact sequences

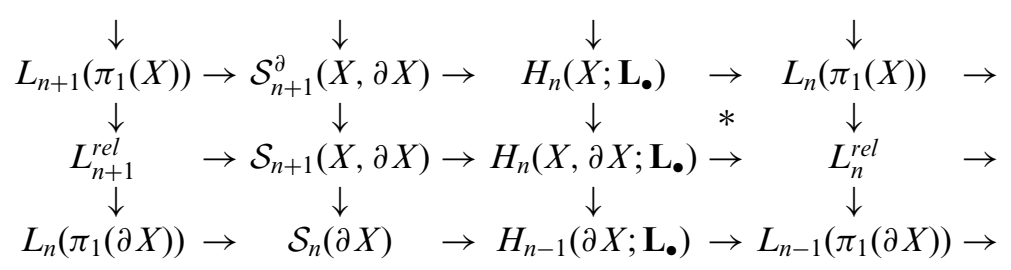

where

$$
L_{*}^{r e l}=L_{*}\left(\pi_{1}(\partial X) \rightarrow \pi_{1}(X)\right) .
$$

It is necessary to remark here that the left parts of the horizontal exact sequences in diagram (2.6) are isomorphic to exact sequences (1.1)-(1.3) (see [14] and [15]).

LEMMA 1. There is a homotopy commutative square of spectra

$$
\begin{array}{ccc}
X_{+} \wedge \mathbf{L}_{\bullet} & \rightarrow & \mathbb{L}\left(\pi_{1}(X)\right) \\
\downarrow & & \downarrow \\
(X / \partial X)_{+} \wedge \mathbf{L}_{\bullet} & \rightarrow & \mathbb{L}\left(\pi_{1}(\partial X) \rightarrow \pi_{1}(X)\right)
\end{array}
$$

such that the square $*$ in diagram (2.6) is obtained by applying $\pi_{n}$ to homotopy commutative square (2.7).

Proof. The result follows from [14] and [15].

We remark that the left vertical column of diagram (2.6) coincides with relative exact sequence (2.2) for the map $\pi_{1}(\partial X) \rightarrow \pi_{1}(X)$.

3. Mixed structures on a bordered manifold. Let us consider a compact topological manifold pair $(X, \partial X)$ of dimension $n$. Let $f:(M, \partial M) \rightarrow(X, \partial X)$ be a normal map of manifold pairs such that the restriction $\left.f\right|_{\partial M}: \partial M \rightarrow \partial X$ is a simple homotopy equivalence. Two normal maps $f_{i}:\left(M_{i}, \partial M_{i}\right) \rightarrow(X, \partial X), i=0,1$, are said to be equivalent if there exists a normal cobordism $F: W \rightarrow X \times I$, where $I=[0,1]$, satisfying the following properties:

(i) $\partial W=M_{0} \cup M_{1} \cup V$ with $V \cap M_{0}=\partial M_{0}, V \cap M_{1}=\partial M_{1}, \partial V=\partial M_{0} \cup \dot{ن} \partial M_{1}$, and $\left.F\right|_{M_{i}}=f_{i}$, for $i=0,1$;

(ii) setting $V=F^{-1}(\partial X \times I)$, the restriction $\left.F\right|_{V}$ is an $s$-cobordism between $\left.F\right|_{\partial M_{i}}=\left.f_{i}\right|_{\partial M_{i}}$, for $i=0,1$.

The set of equivalence classes of such maps is denoted by $\mathcal{T} \mathcal{S}(X, \partial X)$ (see [24, p. 116]). The set $\mathcal{T} \mathcal{S}(X, \partial X)$ has a base point which is represented by a homeomorphism

$$
f:(M, \partial M) \rightarrow(X, \partial X)
$$

If we weaken the equivalence relation on the boundary, then we obtain a natural map

$$
\tau: \mathcal{T} \mathcal{S}(X, \partial X) \rightarrow \mathcal{T}(X, \partial X)
$$


where $\mathcal{T}(X, \partial X)$ is the set of concordance classes of $t$-triangulations of the manifold pair $(X, \partial X)$ (see [15, pp. 553-555]). In a similar way we obtain a further natural map

$$
s: \mathcal{T}^{\partial}(X, \partial X) \rightarrow \mathcal{T} \mathcal{S}(X, \partial X) .
$$

If we weaken the equivalence relation on the manifold $M$ but preserve the equivalence relation on the boundary, then we obtain a natural map

$$
t: \mathcal{S}(X, \partial X) \rightarrow \mathcal{T} \mathcal{S}(X, \partial X) .
$$

According to Wall's book (see [24, p. 116]) and using results of Ranicki on surgery in topological category (see [15] and [16]) we obtain the following exact sequences which include the maps $\tau$, $t$, and $s$, respectively:

$$
\begin{gathered}
\cdots \rightarrow L_{n}\left(\pi_{1}(\partial X)\right) \rightarrow \mathcal{T} \mathcal{S}(X, \partial X) \stackrel{\tau}{\rightarrow} \mathcal{T}(X, \partial X) \rightarrow L_{n-1}\left(\pi_{1}(\partial X)\right), \\
\quad \cdots \rightarrow L_{n+1}\left(\pi_{1}(X)\right) \rightarrow \mathcal{S}(X, \partial X) \stackrel{t}{\rightarrow} \mathcal{T} \mathcal{S}(X, \partial X) \rightarrow L_{n}\left(\pi_{1}(X)\right)
\end{gathered}
$$

and

$$
\cdots \rightarrow \mathcal{S}^{\partial}\left(\partial X \times I, \partial X \times S^{0}\right) \rightarrow \mathcal{T}^{\partial}(X, \partial X) \stackrel{s}{\rightarrow} \mathcal{T} \mathcal{S}(X, \partial X) \rightarrow \mathcal{S}(\partial X) .
$$

We can extend the homotopy commutative diagram of spectra in (2.7) to a biinfinite homotopy commutative diagram (see [11] and [23])

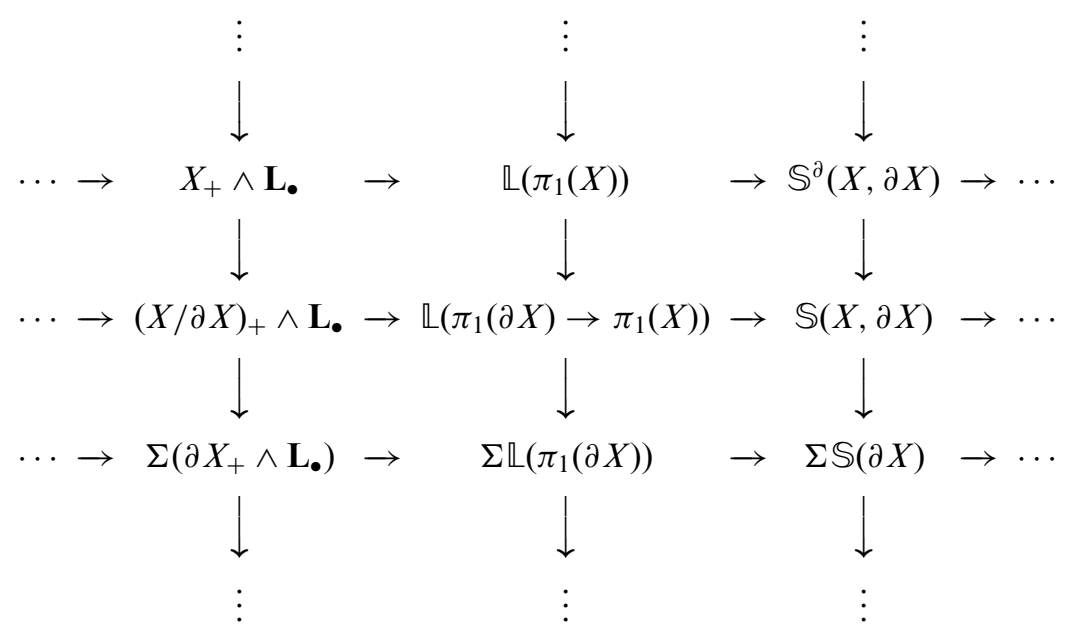

in which the rows and the columns are sequences of cofibrations.

We remark that applying $\pi_{0}$ to homotopy commutative diagram (3.7) yields the bi-infinite commutative diagram of groups in (2.6).

For what follows, we need a technical result proved in [11].

LEMMA 2. Let

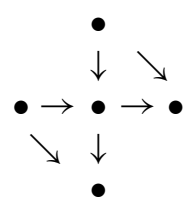


be a homotopy commutative diagram of spectra in which the row and the column are cofibrations. Then the cofibres of the sloping maps are naturally homotopy equivalent.

We define a spectrum $\mathbb{T}(X, \partial X)$ which is the homotopy cofiber of the composition

$$
\mathbb{L}\left(\pi_{1}(X)\right) \rightarrow \mathbb{S}^{\partial}(X, \partial X) \rightarrow \mathbb{S}(X, \partial X)
$$

fitting in diagram (3.7), and denote the homotopy groups of the obtained spectra by

$$
\pi_{n}(\mathbb{T S}(X, \partial X))=\mathcal{T} \mathcal{S}_{n}(X, \partial X) .
$$

PROPOSITION 3. There are the following cofibrations of spectra

$$
\begin{gathered}
\mathbb{L}\left(\pi_{1}(X)\right) \rightarrow \mathbb{S}(X, \partial X) \rightarrow \mathbb{T} \mathbb{S}(X, \partial X), \\
\mathbb{S}(\partial X) \rightarrow \Sigma\left(X_{+} \wedge \mathbf{L}_{\bullet}\right) \rightarrow \mathbb{T} \mathbb{S}(X, \partial X)
\end{gathered}
$$

and

$$
(X / \partial X)_{+} \wedge \mathbf{L}_{\bullet} \rightarrow \Sigma \llbracket\left(\pi_{1}(\partial X)\right) \rightarrow \mathbb{T S}(X, \partial X) .
$$

Proof. The result follows from the definition of the spectrum $\mathbb{T} S(X, \partial X)$ and an application of Lemma 2 to diagram (3.7).

COROLLARY 4. There are the following exact sequences

$$
\begin{gathered}
\cdots \rightarrow L_{n}\left(\pi_{1}(\partial X)\right) \rightarrow \mathcal{T} \mathcal{S}_{n+1}(X, \partial X) \rightarrow H_{n}\left(X, \partial X ; \mathbf{L}_{\bullet}\right) \rightarrow \cdots, \\
\cdots \rightarrow L_{n+1}\left(\pi_{1}(X)\right) \rightarrow \mathcal{S}_{n+1}(X, \partial X) \rightarrow \mathcal{T} \mathcal{S}_{n+1}(X, \partial X) \rightarrow \cdots
\end{gathered}
$$

and

$$
\cdots \rightarrow \mathcal{S}_{n+1}(\partial X) \rightarrow H_{n}\left(X ; \mathbf{L}_{\bullet}\right) \rightarrow \mathcal{T} \mathcal{S}_{n+1}(X, \partial X) \rightarrow \cdots
$$

Proof. The result is obtained by considering the homotopy long exact sequences of cofibrations listed in the statement of Proposition 3.

Proposition 5. There is an isomorphism

$$
\mathcal{T} \mathcal{S}_{n+1}(X, \partial X) \cong \mathcal{T} \mathcal{S}(X, \partial X)
$$

Furthermore, we have a commutative diagram

$$
\begin{aligned}
& \rightarrow L_{n}\left(\pi_{1}(\partial X)\right) \rightarrow \mathcal{T} \mathcal{S}_{n+1}(X, \partial X) \rightarrow H_{n}\left(X, \partial X ; \mathbf{L}_{\bullet}\right) \rightarrow L_{n-1}\left(\pi_{1}(\partial X)\right) \\
& \downarrow=\quad \downarrow \cong \quad \downarrow \cong \quad \downarrow= \\
& \rightarrow L_{n}\left(\pi_{1}(\partial X)\right) \rightarrow \mathcal{T} \mathcal{S}(X, \partial X) \rightarrow \mathcal{T}(X, \partial X) \rightarrow L_{n-1}\left(\pi_{1}(\partial X)\right) \text {. }
\end{aligned}
$$

In particular, there is a group structure on $\mathcal{T} \mathcal{S}(X, \partial X)$ making the bottom sequence in (3.11) an exact sequence of groups.

Proof. The results of Wall (see [24, pp. 116-117]) and Ranicki (see [16, §7.2]) provide commutative diagrams

$$
\begin{aligned}
& H_{n+1}\left(X, \partial X ; \mathbf{L}_{\bullet}\right) \quad \rightarrow L_{n+1}\left(\pi_{1}(\partial X) \rightarrow \pi_{1}(X)\right) \rightarrow L_{n}(\partial X) \\
& \downarrow \cong \quad \downarrow=\quad \downarrow= \\
& \mathcal{T}\left((X, \partial X) \times D^{1} \text { rel } X \times S^{0}\right) \rightarrow L_{n+1}\left(\pi_{1}(\partial X) \rightarrow \pi_{1}(X)\right) \rightarrow L_{n}(\partial X)
\end{aligned}
$$


and

$$
\begin{aligned}
& H_{n}\left(X, \partial X ; \mathbf{L}_{\bullet}\right) \rightarrow L_{n}\left(\pi_{1}(\partial X) \rightarrow \pi_{1}(X)\right) \rightarrow L_{n}(\partial X) \\
& \downarrow \cong \quad \downarrow=\quad \downarrow= \\
& \mathcal{T}(X, \partial X) \rightarrow L_{n}\left(\pi_{1}(\partial X) \rightarrow \pi_{1}(X)\right) \rightarrow L_{n}(\partial X) .
\end{aligned}
$$

The compositions in the upper rows are maps of homotopy groups which are induced by the map of spectra

$$
(X / \partial X)_{+} \wedge \mathbf{L}_{\bullet} \rightarrow \Sigma \mathbb{L}\left(\pi_{1}(\partial X)\right)
$$

This map fits in the extended cofibration sequence (3.9). Then the result in the statement follows from the uniqueness of the cofibration exact sequence (see for example [23]).

REMARK. In a way similar to Proposition 5 we can obtain isomorphisms between other exact sequences of Corollary 4 and exact sequences (3.4) and (3.5), respectively.

Now we describe further algebraic properties of the introduced groups $\mathcal{T} \mathcal{S}_{i}(X, \partial X)$ and their relations to some classical groups in surgery theory for a manifold with boundary.

THEOREM 6. We have the following braids of exact sequences

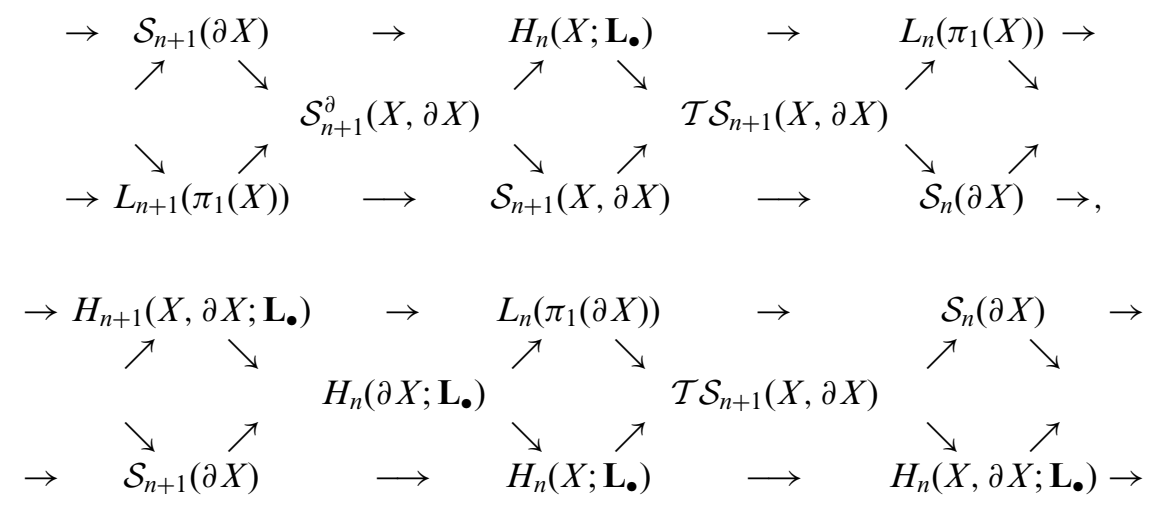

and

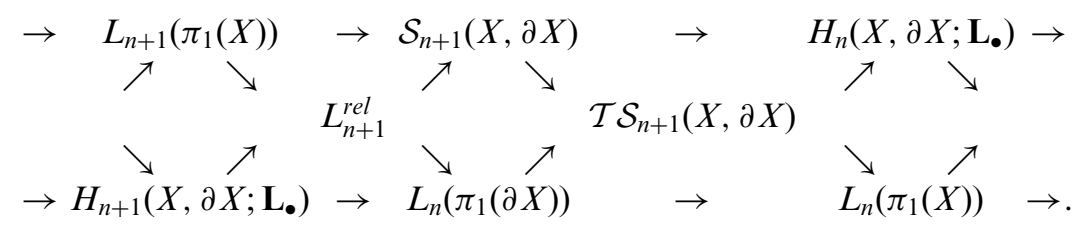

Proof. To prove the result we have to consider the diagonal maps in diagram (3.7), and then apply Lemma 2. First we examine the case of diagram (3.13). From the definition of the spectrum $\mathbb{T S}(X, \partial X)$ we have a homotopy commutative diagram of spectra

$$
\begin{aligned}
& \mathbb{L}\left(\pi_{1}(X)\right) \rightarrow \mathbb{S}^{\partial}(X, \partial X) \rightarrow \Sigma\left(X_{+} \wedge \mathbf{L}_{\bullet}\right) \\
& \begin{array}{ccc}
\downarrow= & \downarrow & \downarrow \\
\left(\pi_{1}(X)\right) \rightarrow \mathbb{S}(X, \partial X) \rightarrow \mathbb{S}(X, \partial X)
\end{array}
\end{aligned}
$$


in which the rows are cofibrations and the right vertical maps are induced by two maps arising from (3.7). The right square in (3.16) is a pull-back since the fibres of the right horizontal maps are naturally homotopy equivalent. The homotopy long exact sequences of maps fitting in right square (3.16) give the braid of exact sequences shown in (3.13).

From diagram (3.7) and cofibration (3.9) we obtain a homotopy commutative diagram of spectra:

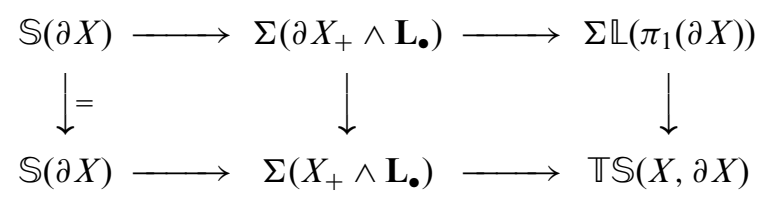

where the right vertical map is induced by two left vertical maps (see [23]). The horizontal rows in (3.17) are cofibrations. Furthermore, the right square is a pullback since the fibers of its horizontal maps are naturally homotopy equivalent. Then the homotopy long exact sequences of maps from this square give rise to the braid of exact sequences shown in (3.14).

From diagram (3.7) and cofibration (3.10) we obtain a homotopy commutative diagram of spectra:

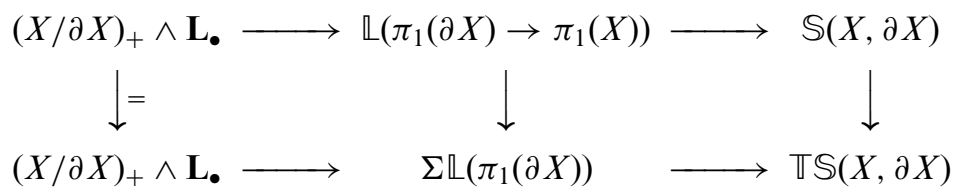

where the right vertical map is induced by two left vertical maps (see [23]). As in the previous case, the right square in (3.18) is a pull-back. Then the homotopy long exact sequences of maps from this square give the braid of exact sequences shown in (3.15). Thus the theorem is completely proved.

4. Examples and applications. In this section we discuss several geometric examples, and apply our results for computing different structure sets of certain manifolds with boundary.

Let $(M, \partial M)$ be a compact topological $n$-manifold with boundary. According to [14], [15, pp. 560-561], and [16, $\S 18]$, there are isomorphisms

$$
\begin{aligned}
& \mathcal{S}^{\partial}\left(M \times \Delta^{k}, \partial\left(M \times \Delta^{k}\right)\right) \longrightarrow \mathcal{S}_{n+k+1}(M), \quad k \geq 0 \\
& \mathcal{S}\left(M \times \Delta^{k}, \partial\left(M \times \Delta^{k}\right)\right) \stackrel{\cong}{\cong} \mathcal{S}_{n+k+1}(M, \partial M), \quad k \geq 0
\end{aligned}
$$

and, in particular,

$$
\begin{aligned}
& \mathcal{S}(M, \partial M) \longrightarrow \mathcal{S}_{n+1}(M, \partial M), \\
& \mathcal{S}^{\partial}(M, \partial M) \underset{\mathcal{S}_{n+1}(M)}{\cong}
\end{aligned}
$$

The first isomorphism in (4.1) also works in the case $\partial M=\emptyset$. 
THEOREM 7. Let $M^{2}$ be a Möbius band with boundary $\partial M^{2}=S^{1}$. For any $n \geq 6$, the structure sets of $M^{2}$ are related by the isomorphisms

$$
\mathcal{S}_{n}^{\partial}\left(M^{2}, \partial M^{2}\right) \cong \mathcal{S}_{n}\left(M^{2}, \partial M^{2}\right)
$$

If $n \geq 5$, then there are isomorphisms

$$
\mathcal{T} \mathcal{S}_{n+1}(M, \partial M) \cong H_{n}\left(M ; \mathbf{L}_{\bullet}\right) \cong \mathbb{Z}, \quad \mathbb{Z}, \quad \mathbb{Z}_{2}, \quad \mathbb{Z}_{2}
$$

for $n=0,1,2,3(\bmod 4)$, respectively. Furthermore, we have

$$
\mathcal{S}_{n}\left(M^{2}, \partial M^{2}\right) \cong \mathbb{Z}
$$

for $n \equiv 1$ or $2(\bmod 4)$, and for $n \equiv 3(\bmod 4)$ there is an exact sequence

$$
0 \rightarrow \mathcal{S}_{n+1}\left(M^{2}, \partial M^{2}\right) \rightarrow \mathbb{Z}_{2} \rightarrow \mathbb{Z}_{2} \rightarrow \mathcal{S}_{n}\left(M^{2}, \partial M^{2}\right) \rightarrow 0
$$

where the middle map arises from the assembly map

$$
\mathbb{Z}_{2} \cong H_{4 k+3}\left(M^{2} ; \mathbf{L}_{\bullet}\right) \longrightarrow L_{4 k+3}\left(\pi_{1}\left(M^{2}\right)\right) \cong L_{4 k+3}\left(\mathbb{Z}^{-}\right) \cong \mathbb{Z}_{2} .
$$

Proof. Consider the exact sequence

$$
\rightarrow \mathcal{S}_{n+1}\left(\partial M^{2}\right) \rightarrow \mathcal{S}_{n+1}^{\partial}\left(M^{2}, \partial M^{2}\right) \rightarrow \mathcal{S}_{n+1}\left(M^{2}, \partial M^{2}\right) \rightarrow \mathcal{S}_{n}\left(\partial M^{2}\right) \rightarrow
$$

which fits in commutative diagram (3.13). Since $\partial M^{2}=S^{1}$ we get

$$
\mathcal{S}^{\partial}\left(S^{1} \times \Delta^{k}, S^{1} \times S^{k-1}\right)=0
$$

for any $k \geq 3$ (see for example [1, p. 403], [22, p. 277], and [24, p. 236]). This result and isomorphism (4.1) imply that $\mathcal{S}_{k+2}\left(\partial M^{2}\right)=\mathcal{S}_{k+2}\left(S^{1}\right)=0$ for $k \geq 3$. Now the first statement of the theorem follows by the exactness of the sequence above. Similarly, from the exact sequence

$$
\cdots \rightarrow \mathcal{S}_{n+1}(\partial M) \rightarrow H_{n}\left(\partial X ; \mathbf{L}_{\bullet}\right) \rightarrow \mathcal{T} \mathcal{S}_{n+1}\left(M^{2}, \partial M^{2}\right) \rightarrow \cdots
$$

fitting in diagram (3.13) we get an isomorphism

$$
H_{n}\left(M ; \mathbf{L}_{\bullet}\right) \cong \mathcal{T} \mathcal{S}_{n+1}\left(M^{2}, \partial M^{2}\right) .
$$

For $n \geq 5$, the homotopy equivalence $M^{2} \simeq S^{1}$ yields

$$
H_{n}\left(M^{2} ; \mathbf{L}_{\bullet}\right) \cong H_{n}\left(S^{1} ; \mathbf{L}_{\bullet}\right) \cong L_{n}\left(\mathbb{Z}^{+}\right)
$$

(see for example [22, p. 277] and [24, p. 236]). By [24, p. 181], we have

$$
L_{n}\left(\mathbb{Z}^{+}\right) \cong \mathbb{Z}, \quad \mathbb{Z}, \quad \mathbb{Z}_{2}, \quad \mathbb{Z}_{2}
$$

and

$$
L_{n}\left(\pi_{1}\left(M^{2}\right)\right) \cong L_{n}\left(\mathbb{Z}^{-}\right) \cong \mathbb{Z}_{2}, \quad 0, \quad \mathbb{Z}_{2}, \quad \mathbb{Z}_{2}
$$

for $n \equiv 0,1,2,3(\bmod 4)$, respectively. Look at the braids of exact sequences in Theorem 6. Their upper and bottom rows, considered as chain complexes, have 
isomorphic homology groups in the corresponding members. For $n \geq 6$, consider the following part of diagram (3.13):

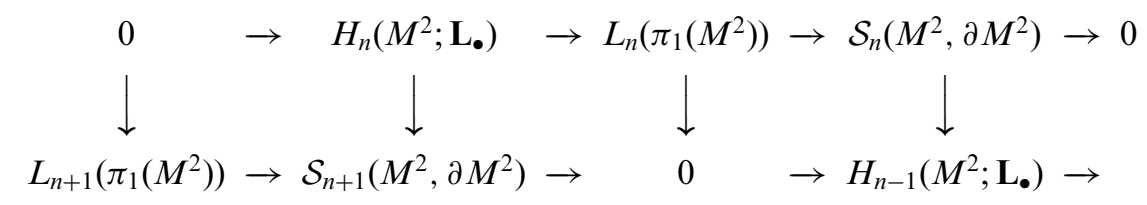

Assume $n=4 k+1$. Then we have $L_{n}\left(\mathbb{Z}^{-}\right) \cong 0, H_{n}\left(M^{2} ; \mathbf{L}_{\bullet}\right) \cong \mathbb{Z}$, and $\mathcal{S}_{n}\left(M^{2}, \partial M^{2}\right)$ in the upper row of the above diagram will be full in homology. To describe the map

$$
\mathbb{Z}_{2} \cong H_{n+1}\left(M^{2} ; \mathbf{L}_{\bullet}\right) \rightarrow L_{n+1}\left(\pi_{1}\left(M^{2}\right)\right) \cong L_{2}\left(\mathbb{Z}^{-}\right) \cong \mathbb{Z}_{2}
$$

we consider a commutative square from diagram (3.15)

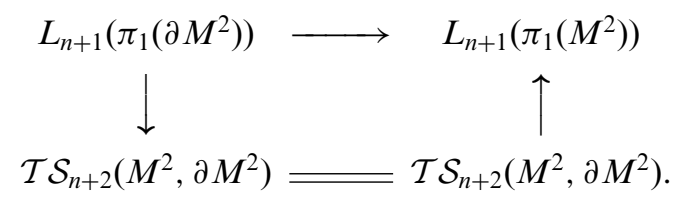

The map in the upper row of (4.5) is induced by the map

$$
i: \mathbb{Z}^{+} \stackrel{\times 2}{\longrightarrow} \mathbb{Z}^{-}
$$

which gives the homomorphism $i_{*}: L_{2}\left(\mathbb{Z}^{+}\right) \cong \mathbb{Z}_{2} \rightarrow L_{2}\left(\mathbb{Z}^{-}\right) \cong \mathbb{Z}_{2}$.

Consider the commutative diagram of groups

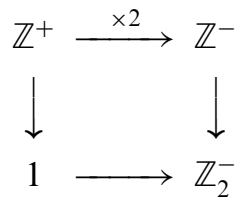

where the vertical maps are the natural projections. Diagram (4.6) induces a commutative diagram of Wall groups

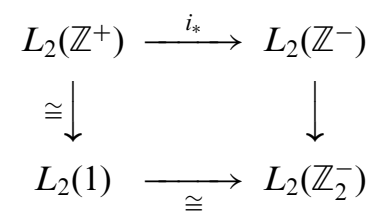

in which the left vertical map is an isomorphism (indeed, the inclusion $1 \rightarrow \mathbb{Z}$ admits a left inverse), and the bottom horizontal map preserves the Arf invariant. Thus the upper horizontal map (and hence the horizontal map in (4.5)) is an isomorphism, too. From diagram (3.13) we have isomorphisms $\mathcal{T} \mathcal{S}_{n+1}\left(M^{2}, \partial M^{2}\right) \cong H_{n}\left(M^{2} ; \mathbf{L}_{\bullet}\right)$ hence for $n=4 k+1$ all the groups in (4.5) are $\mathbb{Z}_{2}$, and all the maps are isomorphisms (since the upper horizontal map is an isomorphism). Consider the commutative diagram arising 
from (3.13)

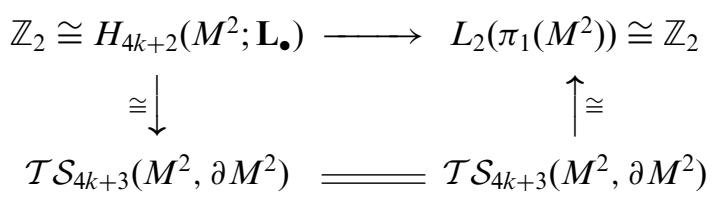

in which the right vertical map is an isomorphism by (4.5). Hence the upper row in (4.7) is an isomorphism, and the map $L_{2}\left(\pi_{1}\left(M^{2}\right)\right) \rightarrow \mathcal{S}_{4 k+2}\left(M^{2}, \partial M^{2}\right)$ in (4.4) is trivial. This implies $\mathcal{S}_{4 k+2}\left(M^{2}, \partial M^{2}\right) \cong H_{4 k+1}\left(M^{2} ; \mathbf{L}_{\bullet}\right) \cong \mathbb{Z}$.

Considering diagram (4.4) for $n=4 k$ we obtain the following diagram.

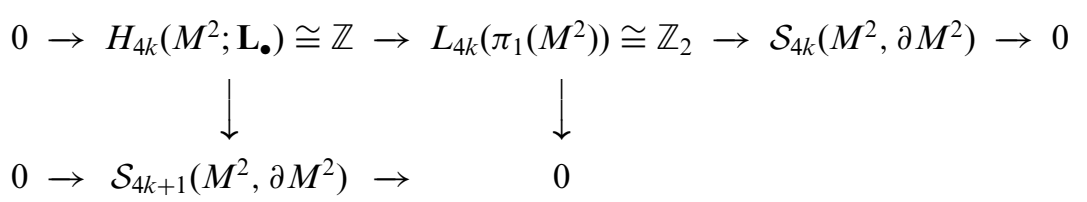

First we remark that the map $i_{*}: L_{0}\left(\mathbb{Z}^{+}\right) \cong \mathbb{Z} \rightarrow L_{0}\left(\mathbb{Z}^{-}\right) \cong \mathbb{Z}_{2}$ is surjective (see [24, p. 189]). Then for $n=4 k-1$ the map $\mathcal{T} \mathcal{S}_{n+2}\left(M^{2}, \partial M^{2}\right) \rightarrow L_{0}\left(\pi_{1}\left(M^{2}\right)\right)$ in (4.5) is surjective, too. From this and a commutative diagram similar to (4.7) it follows that the map $\mathbb{Z} \cong H_{4 k}\left(M^{2} ; \mathbf{L}_{\bullet}\right) \rightarrow L_{0}\left(\pi_{1}\left(M^{2}\right)\right) \cong \mathbb{Z}_{2}$ is surjective with kernel isomorphic to $\mathbb{Z}$. Thus the homology group in the left column of (4.8) is $\mathbb{Z}$ and it is isomorphic to $\mathcal{S}_{4 k+1}\left(M^{2}, \partial M^{2}\right)$. The exact sequence in (4.3) follows from the algebraic surgery exact sequence in the corresponding dimensions.

Of course, one can extend the results of Theorem 7 to any nontrivial (orientable or not) surface with nonvoid boundary. In this case the manifold is homotopy equivalent to a wedge of circles, its boundary is a disjoint union of circles, the fundamental group is a free group, and the Wall groups are direct sums of factors $L_{n}(\mathbb{Z})$.

Proposition 8. Let $X=M^{2} \times T^{k}$ be the product of a Möbius band with a $k$ dimensional torus $T^{k}=S^{1} \times \cdots \times S^{1}, k \geq 1$, with boundary $\partial X=T^{k+1}$.

For $n \geq 6$, we have an isomorphism

$$
\mathcal{S}_{n}^{\partial}(X, \partial X) \cong \mathcal{S}_{n}(X, \partial X) .
$$

If $n \geq 5$, there are also isomorphisms

$$
\mathcal{T} \mathcal{S}_{n+1}(X, \partial X) \cong H_{n}\left(X ; \mathbf{L}_{\bullet}\right) \cong \bigoplus_{0 \leq i \leq k}\left(\begin{array}{l}
k \\
i
\end{array}\right) L_{n-i}(1)
$$

for $n=0,1,2,3(\bmod 4)$, respectively.

Proof. The proof is similar to the first part of the proof of Theorem 7. In this case the group $L_{n}\left(\mathbb{Z}^{k}\right)$ was computed in [24, Theorem 13A.8].

COROLlaRY 9. Let $X=M^{2} \times S^{1}$ be the product of a Möbius band with a circle with boundary $\partial X=T^{2}$. For $k \geq 1$, we have isomorphisms

$$
\mathcal{S}_{4 k+2}^{\partial}(X, \partial X) \cong \mathcal{S}_{4 k+2}(X, \partial X) \cong \mathbb{Z} \oplus \mathbb{Z} .
$$


Proof. The inclusion-induced map $\pi_{1}(\partial X) \rightarrow \pi_{1}(X)$ coincides with the map

$$
i: \mathbb{Z}^{+} \oplus \mathbb{Z}^{+} \rightarrow \mathbb{Z}^{-} \oplus \mathbb{Z}^{+}
$$

which is the identity on the second summand and the multiplication by 2 on the first summand. Here the sign "+" or "-" denotes an orientation of the corresponding group. Consider a commutative diagram of groups

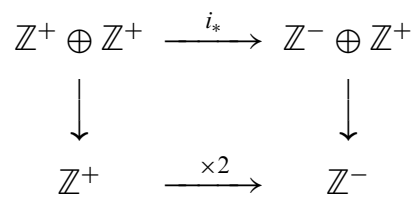

in which the vertical maps are given by projections on the first summand. We obtain the following induced diagram of $L_{n}$-groups

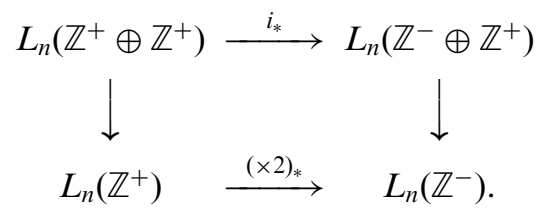

For $n=2$, this diagram has the following form (see $[\mathbf{2 4}, \S 13 \mathrm{~A}]$ )

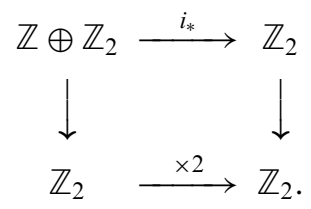

The vertical maps in (4.11) are splitting projections, and the lower horizontal map is an isomorphism as follows from the proof of Theorem 7 . Hence the upper horizontal map in (4.11), which is the map

$$
i_{*}: L_{2}\left(\mathbb{Z}^{+} \oplus \mathbb{Z}^{+}\right) \rightarrow L_{2}\left(\mathbb{Z}^{-} \oplus \mathbb{Z}^{+}\right),
$$

is an epimorphism. The vertical maps in (4.10) give a decomposition of the map

$$
i_{*}: L_{1}\left(\mathbb{Z}^{+} \oplus \mathbb{Z}^{+}\right) \rightarrow L_{1}\left(\mathbb{Z}^{-} \oplus \mathbb{Z}^{+}\right)
$$

into a direct sum of the maps (see [21])

$$
\begin{aligned}
L_{1}\left(\mathbb{Z}^{+}\right) \stackrel{(\times 2)_{*}}{\longrightarrow} L_{1}\left(\mathbb{Z}^{-}\right)=0 \\
L_{0}\left(\mathbb{Z}^{+}\right) \stackrel{(\times 2)_{*}}{\longrightarrow} L_{0}\left(\mathbb{Z}^{-}\right)=\mathbb{Z}_{2} .
\end{aligned}
$$

The map in (4.12) is surjective as follows from the proof of Theorem 7. Hence the map (4.13) is surjective, too. Now from the commutative triangle

$$
\begin{array}{ccc}
\nearrow^{\mathcal{T} \mathcal{S}_{n+1}} & \searrow \\
L_{n}\left(\pi_{1}(\partial X)\right) & \longrightarrow & L_{n}\left(\pi_{1}(X)\right)
\end{array}
$$


fitting in diagram (3.15) it follows that the maps

$$
\mathcal{T} \mathcal{S}_{n+1} \rightarrow L_{n}\left(\pi_{1}(X)\right)
$$

are epimorphisms for $n=1,2(\bmod 4)$. Hence the second exact sequence from Corollary 4 gives the exact sequence

$$
0 \rightarrow \mathcal{S}_{4 k+2}(X, \partial X) \rightarrow \mathcal{T} \mathcal{S}_{4 k+2}(X, \partial X) \stackrel{e p i}{\longrightarrow} L_{1}\left(\pi_{1}(X)\right)=\mathbb{Z}_{2}
$$

Now Proposition 8 yields isomorphisms

$$
\mathcal{T} \mathcal{S}_{4 k+2}(X, \partial X) \cong H_{4 k+1}\left(X, \partial X ; \mathbf{L}_{\bullet}\right) \cong \mathbb{Z} \oplus \mathbb{Z} .
$$

Thus the corollary is proved.

Let us consider a non-trivial $I$-bundle over the real projective space $\mathbb{R} P^{n}, n \geq 5$, and denote by $X^{n+1}$ the total space of this bundle with boundary $\partial X=S^{n}$. In this case, we have

$$
\mathcal{S}_{n+1}(\partial X)=\mathcal{S}_{n+1}\left(S^{n}\right)=0
$$

by a celebrated theorem of Smale, i.e., the Generalised Poincare Conjecture in the topological category. By $\left[\mathbf{1 4}\right.$, p. 310] we have $\mathcal{S}_{n}\left(S^{n}\right)=0$ and

$$
\mathcal{S}_{n+k}\left(S^{n}\right) \cong L_{k-1}(1) \quad \text { for } \quad k \geq 2 .
$$

The space $X$ has the homotopy type of the real projective space $\mathbb{R} P^{n}$. Recall that by [15], [17], and [24, $\S 10]$ there is an isomorphism

$$
H_{n}\left(\mathbb{R} P^{n} ; \mathbf{L}_{\bullet}\right) \cong\left[\mathbb{R} P^{n}, G / T O P\right] .
$$

The results of [19] can be applied to $G / T O P$ as well as to $G / P L$. Thus we get the formula

$$
\left[\mathbb{R} P^{n}, G / T O P\right] \cong \bigoplus_{i=1}^{\infty} H^{4 i-2}\left(\mathbb{R} P^{n} ; \mathbb{Z}_{2}\right) \oplus H^{4 i}\left(\mathbb{R} P^{n} ; \mathbb{Z}\right)
$$

which gives (compare with [19, Lemma 14D.1])

$$
\left[\mathbb{R} P^{2 i+5}, G / T O P\right] \cong\left[\mathbb{R} P^{2 i+4}, G / T O P\right] \cong \mathbb{Z}_{2} \oplus \mathbb{Z}_{2} \bigoplus_{j=1}^{i} \mathbb{Z}_{2} .
$$

The third exact sequence of Corollary 4 provides isomorphisms

$$
H_{i}\left(X ; \mathbf{L}_{\bullet}\right) \cong \mathcal{T S}_{i+1}(X, \partial X) \text { for } \quad i=n, n+1
$$

and the exact sequence

$$
0 \rightarrow H_{m}\left(X ; \mathbf{L}_{\bullet}\right) \rightarrow \mathcal{T} \mathcal{S}_{m+1}(X, \partial X) \rightarrow L_{2 k}(1) \rightarrow H_{m-1}\left(X ; \mathbf{L}_{\bullet}\right) \rightarrow \mathcal{T} \mathcal{S}_{m}(X, \partial X) \rightarrow 0
$$

where $m=n+2 k+1, k \geq 1$. 
Let $K \subset S^{3}$ be a knot, that is, the image of a locally flat embedding of the circle $S^{1}$ in the standard 3-sphere $S^{3}$. Denote by $C$ the complement of a tubular neighborhood of $K$ in $S^{3}$.

Proposition 10. For $n \geq 7$, we have isomorphisms

$$
\mathcal{T} \mathcal{S}_{n}(C, \partial C) \cong 0, \quad \mathbb{Z}, \quad 0, \quad \mathbb{Z}_{2} \quad \text { for } \quad n=0,1,2,3 \quad(\bmod 4) .
$$

Proof. According to [1] we have

$$
\mathcal{S}^{\partial}(C, \partial C)=0 \text { for } n \geq 6 .
$$

For $n \geq 6$, the braid of exact sequences (3.13) provides an isomorphism

$$
\mathcal{S}_{n+1}(C, \partial C) \stackrel{\cong}{\rightarrow} \mathcal{S}_{n}(\partial C)
$$

and the short exact sequence

$$
0 \rightarrow \mathcal{S}_{n+1}(C, \partial C) \rightarrow \mathcal{T} \mathcal{S}_{n+1}(C, \partial C) \rightarrow L_{n}\left(\pi_{1}(C)\right) \rightarrow 0 .
$$

But we have

$$
\mathcal{S}_{n}(\partial C)=\mathcal{S}_{n}\left(T^{2}\right)=0
$$

for every $n \geq 6$, and

$$
L_{n}\left(\pi_{1}(C)\right) \cong L_{n}(\mathbb{Z})=\mathbb{Z}, \quad 0, \quad \mathbb{Z}_{2}, \quad 0 \quad \text { for } \quad n=0,1,2,3 \quad(\bmod 4)
$$

(compare with [1]). Now the statement of the theorem follows from (4.15).

The last example deals with Haken manifolds so we recall the definition to make the reading self-contained (for more details see [6], [7], [8, p. 235], [9, p. 63], [10, 6 , p. 215], and [18]). Let $F$ be a compact proper surface embedded in a compact connected 3-manifold $M$. We call $F$ a $t w o$-sided surface in $M$ if $F$ cuts a regular neighborhood of $F$ into two pieces, i.e., the normal bundle of $F$ is oriented. Assuming that $M$ is oriented, this is equivalent to the condition that $F$ is oriented. A two-sided surface $F$ in $M$ is incompressible if every simple curve on $F$ which bounds a disc in $M$ with interior disjoint from $F$ also bounds a disc on $F$, i.e., the homomorphism $\pi_{1}(F) \rightarrow \pi_{1}(M-\operatorname{int} F)$ is injective. A 3-manifold $M$ is said to be irreducible if every 2-sphere in $M$ bounds a 3-ball in $M$. A compact connected oriented 3-manifold $M$ is called a Haken manifold if it is irreducible and contains a two-sided incompressible surface (whose boundary, if any, is on $\partial M$ ) which is not a 2 -sphere.

Proposition 11. Let $M$ be a compact oriented connected Haken 3-manifold with non-empty boundary $\partial$ M. For $n \geq 6$, we have isomorphisms

$$
\mathcal{T} \mathcal{S}_{n+1}(M, \partial M) \cong \mathcal{S}_{n}(\partial M) \oplus L_{n}\left(\pi_{1}(M)\right) .
$$

Proof. According to [18, Theorem 1.1] we have $\mathcal{S}_{n}^{\partial}(M, \partial M)=0$ for $n \geq 6$. For $n \geq 6$, the braid of exact sequences (3.13) provides an isomorphism

$$
\mathcal{S}_{n+1}(M, \partial M) \stackrel{\cong}{\rightrightarrows} \mathcal{S}_{n}(\partial M)
$$


and the short exact sequence

$$
0 \rightarrow \mathcal{S}_{n+1}(M, \partial M) \rightarrow \mathcal{T} \mathcal{S}_{n+1}(M, \partial M) \rightarrow L_{n}\left(\pi_{1}(C)\right) \rightarrow 0 .
$$

The commutative triangle fitting in (3.13)

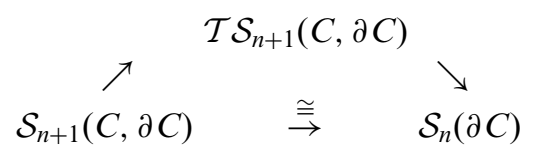

implies that exact sequence (4.16) splits. From this the statement of the proposition follows.

The next result is a version of $\pi-\pi$-theorem for structure sets $\mathcal{T} \mathcal{S}_{n}(M, \partial M)$.

Proposition 12. Let $M$ be a compact manifold of dimension $n \geq 6$ with boundary $\partial M$. Suppose that the inclusion-induced homomorphism $\pi_{1}(\partial M) \rightarrow \pi_{1}(M)$ is bijective. For $n \geq 6$, we have isomorphisms

$$
\mathcal{T} \mathcal{S}_{n+1}(M, \partial M) \cong \mathcal{S}_{n}(M, \partial M) \oplus L_{n}\left(\pi_{1}(M)\right) \cong H_{n}\left(M, \partial M ; \mathbf{L}_{\bullet}\right) \oplus L_{n}\left(\pi_{1}(M)\right) .
$$

Proof. The proof is similar to the proof of Proposition 11. But in this case it is necessary to consider commutative diagram (3.15) instead of (3.13).

\section{REFERENCES}

1. C. S. Aravinda, F. T. Farrell and S. K. Roushon, Surgery groups of knot and link complements, Bull. London Math. Soc. 29 (1997), 400-406.

2. A. Bak and Yu. V. Muranov, Splitting along submanifolds and $L$-spectra, in Contemporary Mathematics and Applications. Topology, Calculus, and Related Questions 1 (2003), 3-18.

3. A. Bak and Yu. V. Muranov, Normal invariants of manifold pairs and assembly maps, in preparation.

4. A. Cavicchioli, Yu. V. Muranov and D. Repovš, Algebraic properties of decorated splitting obstruction groups, Boll. Un. Mat. Ital. 4-B (8) (2001), 647-675.

5. A. Cavicchioli, Yu. V. Muranov and F. Spaggiari, Relative groups in surgery theory, Bull. Belgian Math. Soc.-Simon Stevin 12 (2005), 109-135.

6. W. Haken, Theorie der normal flächen, Acta Math. 105 (1961), 245-375.

7. J. Hempel, 3-Manifolds, Ann. of Math. Studies 86 (Princeton Univ. Press, Princeton, N.J. 1976).

8. A. Kawauchi, A survey of knot theory (Birkhäuser, 1996).

9. C. MacLachlan and A. W. Reid, The arithmetic of hyperbolic 3-manifolds, Graduate Texts in Math. No. 219 (Springer Verlag, 2003). 2003).

10. S. Matveev, Algorithmic topology and classification of 3-manifolds, (Springer Verlag,

11. Yu. V. Muranov, Obstruction groups to splitting and quadratic extensions of antistructures, Izvestiya RAN: Ser. Mat. 59 (6) (1995), 107-132 (in Russian); English transl. in Izvestiya Math. 59 (6) (1995), 1207-1232.

12. Yu. V. Muranov and D. Repovš, Groups of obstructions to surgery and splitting for a manifold pair, Mat. Sbornik 188 (3) (1997), 127-142 (in Russian); English transl. in Russian Acad. Sci. Sb. Math. 188 (3) (1997), 449-463.

13. Yu. V. Muranov, D. Repovš and F. Spaggiari, Surgery on triples of manifolds, Mat. Sbornik 194 (8) (2003), 1251-1271. 
14. A. A. Ranicki, The total surgery obstruction, Lecture Notes in Mathematics No. 763 (Springer-Verlag, 1979).

15. A. A. Ranicki, Exact sequences in the algebraic theory of surgery, Math. Notes No. 26 (Princeton University Press, Princeton, N.J., 1981).

16. A. A. Ranicki, Algebraic $L$-theory and topological manifolds, Cambridge Tracts in Math. (Cambridge University Press, 1992).

17. A. A. Ranicki, An introduction to algebraic surgery, in: Surveys on surgery theory (S. Cappell, A. A. Ranicki, and J. Rosenberg, eds.) 2 (2001), Annals of Math. Studies No. 149 (Princeton University Press, Princeton, N.J.), 81-163.

18. S. K. Roushon, Vanishing structure set of Haken 3-manifolds, Math. Ann. 318 (2000), 609-620.

19. Yu. B. Rudyak, On normal invariants of certain manifolds, Mat. Zametki 16 (1974), $763-769$.

20. B. Ruini and F. Spaggiari, On the computation of $L$-groups and natural maps, $A b h$. Math. Sem. Univ. Hamburg 72 (2002), 297-308.

21. J. L. Shaneson, Wall's surgery obstruction groups for $G \times \mathbb{Z}$, Ann. of Math. 90 (1969), $296-334$.

22. C. W. Stark, Surgery theory and infinite fundamental groups, in Surveys on surgery theory (S. Cappell, A. A. Ranicki, and J. Rosenberg, eds.) 1 (2000), Annals of Math. Studies No. 145 (Princeton University Press, Princeton, N.J.), 275-305.

23. R. M. Switzer, Algebraic topology-homotopy and homology (Springer Verlag, 1975).

24. C. T. C. Wall, Surgery on compact manifolds (Academic Press, 1970) Second Edition (A. A. Ranicki ed.) (Amer. Math. Soc., Providence, R.I. 1999). 